\title{
Liderazgo del Directivo para Mejorar Relaciones Interpersonales en Personal de Organizaciones Educativas
}

\author{
Autor: César Orlando Becerra Ramírez \\ Universidad Experimental "Rafael María Baralt", UNERMB \\ cesarbecerra70@gmail.com \\ Trujillo, Venezuela
}

\section{Resumen}

El artículo contiene una reflexión teórica en torno al Liderazgo del Directivo para mejorar las Relaciones Interpersonales en personal de organizaciones Educativas. Por lo tanto, su objetivo es describir la importancia del Liderazgo del Directivo para Mejorar las Relaciones Interpersonales en personal de las instituciones educativas. Desde esta premisa, el artículo constituye un aporte para lectores interesados en el Liderazgo y las Relaciones Interpersonales, adoptando como metodología la investigación documental, de tipo argumentativa e informativa. Obteniendo como conclusiones: El perfil del líder es en mayor grado humanista; El Liderazgo del Directivo, es un elemento que debe estar presente en toda organización; Se observa el desarrollo y fortalecimiento de las relaciones interpersonales sanas dentro de las instituciones.

Palabras clave: liderazgo; directivo; relaciones interpersonales. 


\title{
Executive Leadership to Improve Personal Relationships in Educational Organizations
}

\begin{abstract}
The article contains a theoretical reflection on the Leadership Board to improve interpersonal relations in organizations Educational personnel. So your goal is to describe the importance of the Executive Leadership to Improve Interpersonal Relations staff of educational institutions. From this premise, the article is a contribution to readers interested in leadership and Relationships, taking as documentary research methodology, argumentative and informative type. Getting as conclusions: The profile of the leader is more humanistic level; The Leadership Board is an element that must be present in every organization; The development and strengthening of healthy interpersonal relationships within institutions is observed.
\end{abstract}

Keywords: leadership; executive; relationships. 


\section{Introducción}

Los retos que enfrenta la educación en todo el mundo son enormes, dándole a esta alta prioridad en el desarrollo de los países, las sociedades, las organizaciones. Es tan grande e imprevisible el impacto de estos cambios, que nadie de momento puede saber con absoluta certeza hacia dónde vamos y cuál será su huella sobre las empresas, las comunidades, las instituciones públicas, privadas y nuestras propias vidas.

En efecto, la visión percibida en general por los trabajadores de su jefe es que ordenan, mandan, deciden, dicen lo que se debe hacer, imponen criterios, distribuyen el trabajo, controlan y supervisan las tareas. Por esto, la preocupación de los directivos debería estar centrada en crear una imagen tal, que sus subordinados lo catalogaran como un colaborador más, orientador, escucha de su gente, generador de confianza; aceptado naturalmente por el grupo, buen comunicador, persona que apoye y ayude, transmita seguridad.

Debido a las razones expuestas, el verdadero líder trabaja para ser aceptado por el personal a su cargo por su carisma, su servicio a un equipo al cual ayuda y orienta para cumplir con las metas prefijadas que se han negociado previamente. El líder es el respaldo del equipo, potencia a las personas para desarrollar sus inquietudes, iniciativas y creatividad. Además, fomenta la responsabilidad, las relaciones interpersonales, el espíritu de equipo, el desarrollo personal y especialmente, es el artesano de la creación de un espíritu de pertenencia en sus colaboradores.

Dentro de esta óptica, para que este cambio fundamental ocurra, es preciso contar con directores capaces de asumir el liderazgo en el proceso de gestión siendo una tarea pendiente para los líderes educativos de la escuela. Entretanto, la Organización de las Naciones Unidas para la Ciencia, la Educación y la Cultura. UNESCO, (2013) en el taller de Liderazgo Directivo Escolar en América Latina y el Caribe, celebrado en Santiago de Chile 
expusieron "la acción de los directivos tiene una poderosa repercusión entre los otros actores educacionales". Dicho en otras palabras, en la actualidad se han vuelto un blanco de políticas públicas, apostando muchas reformas y cambios educativos a las potencialidades de estos agentes, para producir mejora dentro de las escuelas.

En otras palabras, se observa la importancia para esta institución de tal figura, dándole el crédito loable por su incansable labor a favor de los cambios y reformas que emergen en distintos ámbitos y especialmente en el sector educativo, por tanto, se hace necesario fortalecer la capacidad de liderazgo de los directivos para innovar efectivamente la cultura de las instituciones escolares. Respecto, al liderazgo directivo Alfonso (2001), señala:

Quien enfrenta hoy el reto de dirigir una institución educativa, más que un administrador tendrá necesariamente que ser un líder educativo, para lograr resultados óptimos en las condiciones en que vivimos, porque no basta con reunir los requisitos necesarios para el cargo, ni acumular una larga trayectoria docente y directiva, sino también una visión que trascienda los parámetros del centro educativo (pág. 13).

En efecto, tal aseveración es de reflexionar por tantas experiencias observadas en la cotidianidad donde el currículo académico del director es bueno, más no se compagina con la filosofía de la institución, entendiendo el liderazgo como una nueva ética de dirección capaz de movilizar todos los recursos de la organización, especialmente los potenciales humanos, en el logro de la misión.

Ahora bien, es importante la existencia del liderazgo pues en toda organización éste debe de estar para tener una planeación, control y procedimientos adecuados a la vez, permite tener un equipo de trabajo motivado porque el propósito fundamental del liderazgo es el de producir un cambio útil, especialmente no cuantitativo. Lo anterior se aúna a, lo considerado por Hunter, (2001), "liderar consiste en servir a los demás pues 
un buen líder ha de estar pendiente de las necesidades de sus subordinados para atender a sus legítimas necesidades, ayudándoles a cumplir sus aspiraciones, aprovechando sus capacidades al máximo" (pág. 31).

Esta situación evidencia que, hoy en día las organizaciones educativas invitan al compromiso de directivos para mejorar las relaciones y lograr las metas trazadas, incorporando al equipo de trabajo en todas las actividades dentro y fuera de la institución, porque en la humanidad los sujetos se hallan en mutua dependencia y reciprocidad. El contacto equitativo y la comunicación son fundamentales, tanto para el individuo como para el grupo de tal manera que sin ellos, la persona declinaría y el grupo dejaría de funcionar.

En ese orden de ideas, Zaldívar (2004), considera "todas las personas establecen relaciones a lo largo de la vida como las que se dan con padres, hijos, amigos, compañeros de estudio, trabajo. A través de ellas, intercambian formas de sentir, comparten necesidades, intereses y afectos" (pág. 54). A estas relaciones, se les conoce como relaciones interpersonales. Éstas se basan, en la interacción no sólo con la persona sino con sus propias experiencias, sentimientos, valores, conocimientos y formas de vida, totalmente diferentes unos de otros.

Por su parte, García, (2003), indica "las relaciones interpersonales se plantean en términos de las distintas posturas que adoptan las personas con respecto a otras próximas y agrega éstas se refieren, a las actitudes, la red de interacciones que mantienen los agentes personales" (pág. 2). De ahí, pueden presentarse actitudes positivas como: cooperación, acogida, autonomía, participación, satisfacción; pero también se puede observar actitudes de reserva, competitividad, absentismo, intolerancia, frustración, que producen una corriente interna, explícita o no, de deseos, aspiraciones e intereses corporativos y personales.

En tal sentido, según Gardner (1983) "el personal de cada institución debe compartir necesidades, intereses, enmarcados en un contexto 
interpersonal interactuando entre sí y los empleados, utilizando su inteligencia interpersonal que es la habilidad para interactuar con otros comprendiendo sus emociones y controlando las propias". En ese orden de ideas, se requiere producir respuestas a un mundo en constantes desafíos, nuevos retos de grandes magnitudes, haciéndose necesario que el liderazgo del directivo posea cualidades para orientar, dirigir, tomar decisiones, relacionarse, lograr resultados; porque de su gestión depende el éxito de la institución educativa, así como de las sanas relaciones interpersonales del grupo que está dirigiendo.

Amparados al orden del discurso, es notable la relevancia atribuida hasta ahora a la figura del director en las instituciones educativas, debido a esto el autor colocó por título a la indagación "Liderazgo del Directivo para Mejorar Relaciones Interpersonales en personal de Organizaciones Educativas", pretendiendo responder a la interrogante ¿Cuáles conocimientos de liderazgo posee el directivo para mejorar las relaciones interpersonales en el personal de organizaciones educativas?

Con esto, se quiere hacer una descripción detallada de las variables en cuestión, en busca de dar respuesta al objetivo general Describir la importancia del Liderazgo del Directivo para Mejorar las Relaciones Interpersonales en personal de las Organizaciones Educativas, para lo cual se aplicó una metodología de tipo documental, obtenida de fuentes secundarias, con la posible conexión de opiniones entre varios autores y las del investigador. Por esto, su premisa requiere reunir, interpretar y evaluar datos e ideas en forma ecuánime y decorosa. 


\section{Liderazgo}

Las consideraciones teóricas, fueros recogidas de fuentes de varios autores tomando en cuenta la notabilidad para el liderazgo del directivo y las relaciones interpersonales en el personal de las organizaciones educativas, se incluyen las que a juicio del autor representan significatividad y relevancia para el estudio mostrado. Cada vez es más común, que los directores de las organizaciones educativas muestren un creciente interés por conocer los efectos desplegados sobre su personal, tanto en la estructura como en los procesos que se dan en la organización, toda vez que afectan fundamentalmente, la conducta de los que en ella laboran.

La generalidad de los directores en una empresa dirige más de la cuenta, por tanto, se piensa es preciso emplear el término líder en lugar del de director, al respecto Hunter, (et al) señala "inspiran con una visión clara cómo se deben hacer las cosas, éstos no dirigen, sino que guían y apoyan su capacidad de liderazgo sobre la confianza y el respeto" (pág. 40).

Visto desde esta perspectiva, el líder está abocado para el logro de los objetivos organizacionales haciéndolo de una forma planificada, responsable, organizada, es recomendable que sea un proceso compartido con los miembros del equipo y los colaboradores logrando de esta manera, diferentes y variadas satisfacciones para todos, aumentado su productividad personal y competitiva. Por tanto, si el liderazgo es un arte de influir, su característica principal como valor está en las relaciones, es decir, son las relaciones humanas el objeto fundamental del ejercicio del liderazgo. Ahora bien, según Matos (2009) son tres las funciones en el que el líder debe ser un experto:

1. Dirigir equipos de trabajo: Esto se logra planificando una agenda, brindando a todos, la oportunidad para participar, sondear de manera adecuada, abreviando y cristalizando el consenso, es decir, buscando la participación de todos y delegar funciones en aspectos, donde la ayuda de los miembros de la organización sea oportuna y efectiva. 
2. Preparar a otros en el trabajo: Es el proceso diario de ayudar a los otros, a que reconozcan las ocasiones para retocar su desempeño. Un buen preparador observa lo hecho por la gente, le muestra los problemas o ineficiencias de sus métodos, ofrece sugerencias para mejorarlos y ayuda a utilizarlos de una manera positiva.

3. Asesorar: Los asesores efectivos mantienen la intimidad, escuchan de una manera atenta y solidaria los sentimientos y las circunstancias de los demás, les ayudan a determinar lo que tienen que hacer, lo cual incluye la búsqueda de ayuda profesional (pág. 5).

Desde esta premisa, el directivo como líder debe reconocer si en la empresa existe un sistema social. Para actuar en este medio benéfico, primero el administrador debe tener conocimiento de los fenómenos de la conducta humana y abordarlos. Esto último implica cierta comprensión de las necesidades y las motivaciones humanas, y capacidad perceptiva para analizar al individuo. Una vez que haya aprendido todo acerca de la conducta humana, puede orientar el rumbo del liderazgo mediante el incentivo y la motivación.

En este sentido, según Alvarado, (2003), "el liderazgo es la acción de mover a la gente en una dirección por medios no coercitivos" (pág. 48) así, en la administración se constituye en la función de conducir, guiar, dirigir a los colaboradores en base a la fuerza de las ideas, del carácter, del talento, la voluntad y la habilidad administrativa hacia el logro de los objetivos institucionales pre establecidos.

\subsection{Liderazgo y Dirección}

El liderazgo constituye uno de los temas administrativos más investigados y estudiados en las últimas décadas. Al respecto, Calero, (2005), señala "tener actitudes tradicionales de jefe formal es limitante e inconveniente 
para la organización" (pág. 284). Lo distintivo en este caso, es para ejercer de mejor modo la dirección de una institución es deseable hacerlo con características y cualidades del liderazgo, es decir con una administración renovada donde el director no es un jefe clásico sino un líder, un director que asuma nuevas mentalidades, nuevas actitudes, para generar espiritualidad en toda la organización.

La exposición anterior, permite destacar algunas precisiones importantes: La capacidad para guiar y dirigir con efectividad es uno de los requisitos clave para ser administrador excelente; La esencia del liderazgo es el seguimiento, el deseo de las personas por seguir a alguien en este caso al líder, a quien perciben como medio para lograr sus propios deseos, motivos y necesidades. Considerando lo expuesto se puede decir, el liderazgo a diferencia del simple ejercicio del poder, es inseparable de las necesidades y objetivos de los que los siguen y alcanzan, estimulándose mutuamente, niveles de motivación y moralidad más elevados.

Asimismo, el líder despierta la confianza en sus seguidores, se sienten más capaces de alcanzar los objetivos que ellos y él comparten. Como respuesta a esta situación, en su afán por gerenciar adecuadamente el líder entre sus tareas debe propiciar un clima de armonía en la institución, mejorando las relaciones interpersonales entre sus miembros.

\subsection{Relaciones Interpersonales}

En la sociedad actual, cada vez se da con mayor asiduidad el creciente uso de los medios de comunicación audiovisuales de masas y aunque parezca paradójico, existe cada vez menos comunicación interpersonal. En tal sentido, Chiavenato, (2000), afirma; “... las relaciones humanas dan origen a determinado clima en las relaciones interpersonales, entendiéndose por clima la atmósfera psicológica, característica existente en cada organización y las distingue de las otras e influye en el comportamiento de las personas" (pág. 
320). O sea, el desarrollo y fortalecimiento de las relaciones interpersonales sanas dentro de las instituciones son de vital importancia para la propia existencia y se logra a través de todos los actores en la vida cotidiana.

Al respecto, Albert (2004), define las relaciones interpersonales como:

"Un elemento irreducible de la realidad". Se refiere además, a los sentimientos interpersonales, las transacciones emocionales $\mathrm{O}$ afectivas $\mathrm{El}$ amor es tan real como el odio o la soledad... (pág. 60), es decir los sentimientos interpersonales están vinculados a la experiencia intima de los individuos, su alegría y sus disgustos, su amor, su miedo, su aburrimiento, etc.

De todo lo expuesto anteriormente, se puede afirmar que los sentimientos influyen de manera determinante en las relaciones interpersonales, y están vinculados a las experiencias íntimas de los individuos. Entretanto, éstas son precisas para lograr los objetivos organizacionales, pues mediante los contactos que establezcan entre sí, las personas procurarán satisfacer las necesidades del contacto social, y solo si estás compensada, podrán colaborar eficazmente con las metas planteadas.

\subsection{Procesos Sociales que Intervienen en las Relaciones Interpersonales}

2.3.1. Conflicto. El conflicto define al "conjunto de dos o más hipotéticas situaciones que son excluyentes", esto quiere decir, no pueden darse en forma simultánea. Por lo tanto, cuando surge un conflicto se produce un enfrentamiento, una pelea, una lucha o una discusión, donde una de las partes intervinientes intenta imponerse a la otra, el conflicto es un desacuerdo entre las partes que intervienen en el proceso de relaciones interpersonales.

2.3.2. Comunicación efectiva. La comunicación efectiva es aquella que conlleva a existir una constante interacción entre sus miembros, evitando de esta forma el deterioro de las relaciones interpersonales; a todo ello hace 
referencia Ribeiro (2003), cuando señala, "La comunicación es la más básica y vital de todas las necesidades, después de la supervivencia física" (pág. 11).

En este sentido, las organizaciones tienen que asegurar una comunicación efectiva, consciente e intencionada, a fin de lograr entre sus miembros respuestas satisfactorias, tomando en cuenta que el ser humano experimenta muchas emociones, las cuales se manifiestan en mayor cantidad en su rostro.

2.3.3. Liderazgo. El liderazgo es uno de los procesos de mayor importancia en la vida de cualquier grupo humano; ejerce gran influencia sobre las actividades que realizan los individuos, de manera de lograr objetivos en determinadas situaciones. El liderazgo es de carácter situacional. Al respecto, Robbins, (2004), establece "es la habilidad para influir en un grupo y lograr la realización de metas" (pág. 413).

De acuerdo a esta definición, el liderazgo tolera básicamente el intento de influir en la conducta de las demás personas, siempre con la finalidad de dirigir dicho comportamiento al alcance de las metas y el proceso de dirección, éste se lleva a cabo a través de la comunicación. El liderazgo traslada al grupo hacia sus objetivos, dependiendo de un momento o situación determinada de la misma historia del grupo.

\section{Metodología}

Los aspectos metodológicos, forman parte del paisaje en el recorrido de la investigación, tomando en cuenta el proceso de la indagación para obtener información relevante, fidedigna e imparcial, para extender, verificar, corregir o aplicar el conocimiento, es decir, consiste en la búsqueda de la verdad: es decir acerca de la realidad que se pretende conocer o de aquello que significa descubrir o descorrer el manto de algo a investigar.

En orden a estas premisas, el estudio se corresponde con una investigación de tipo documental, según Ortiz y García, (2002), "la 
investigación documental es la presentación de un escrito formal que sigue una metodología reconocida consiste primordialmente, en la presentación selectiva de lo que expertos ya han dicho o escrito sobre un tema determinado" (pág. 56). Debido a esto, la cantidad de información que se genera en todo el mundo es inmensa. El adquirir estos conocimientos se hace con frecuencia mediante la investigación documental realizada en fuentes secundarias. Además, puede presentar la posible conexión de ideas entre varios autores y las ideas del investigador. Su preparación requiere que éste reúna, interprete, evalúe y reporte datos e ideas en forma imparcial, honesta y clara.

\subsection{Tipo de Investigación}

Existen dos tipos de investigación documental según Ortiz y García (et.al) "argumentativa e informativa" (pág. 56). En este caso se considera una indagación: Informativa (expositiva). Este escrito es básicamente una panorámica acerca de la información relevante de diversas fuentes confiables sobre un tema específico, sin tratar de aprobar u objetar alguna idea o postura. Toda la información presentada se basa en lo que se ha encontrado en las fuentes.

En este sentido, la contribución del investigador radica en analizar y seleccionar de esta información aquello que es relevante para su investigación para posteriormente, organizar la información, cubrir todo el tema, sintetizar las ideas y después, presentarlas en un reporte final que a la vez, sea fluido y esté claramente escrito.

\section{Consideraciones Finales}

En este apartado del trabajo, se pueden visualizar algunas conclusiones a las que se ha llegado, luego de la consulta de las fuentes bibliográficas pertinentes al tema con el propósito de obtener una visión más profunda de la temática tratada y aportar razones para que el Liderazgo del Directivo mejore 
las relaciones interpersonales en los contextos de las organizaciones educativas, por tanto, esto nos lleva a exponer algunas consideraciones:

El perfil del líder es en mayor grado humanista, porque se busca la interrelación de los colaboradores con el logro de los objetivos de una manera eficiente, una personalidad adecuada mostrando equilibrio emocional, capacitación y formación acorde a su cargo, ejerciendo el mando sin autoritarismo, es motivador, optimista en los resultados.

El líder actúa para ayudar a un grupo a lograr sus objetivos, con la aplicación máxima de sus capacidades, pero nunca pierde su propia identidad; toma su lugar frente al grupo facilitando su progreso e inspirándolos para cumplir las metas de la organización.

El Liderazgo del Directivo, es un elemento que debe estar presente en toda organización, y en un organismo educacional es uno de los semilleros fundamentales, porque abona el camino para que éste de buenos frutos y al saborearlos emerjan ideas, situaciones orientadas al esfuerzo de todos para el alcance de las metas propuestas, o sea, es el que compromete a la gente a la acción.

Una de las principales destrezas del Líder en la dirección debe ser, utilizar y manejar de manera acertada y efectiva sus habilidades comunicativas, en dependencia de los diferentes contextos sociales en los que se desenvuelve, para el éxito de las buenas relaciones interpersonales.

Es de hacer notar, la existencia del fenómeno de la incomunicación porque existe dentro de la familia como en otros entornos formales e informales. Y también, se da en todas las capas sociales y edades. Es por ello, la falta de empatía (ponerse en situación del otro), de tiempo para escuchar, de fluidez comunicativa es un verdadero problema.

De allí que recientemente, se observa el desarrollo y fortalecimiento de las relaciones interpersonales sanas dentro de las instituciones, son de vital importancia para la propia existencia y se logra a través de todos los actores 
en la vida cotidiana de la organización. Cuestión ésta que no es fácil, pues es natural la existencia de conflictos dentro de las instituciones, porque todas las personas no son iguales por lo tanto están propensos a desacuerdos en su trabajo diario.

En las organizaciones educativas debe existir relaciones interpersonales efectivas entre directores, personal docente, administrativo, alumno, obrero y padres y representantes, que ayuden al desenvolvimiento y al desarrollo de las tareas asignadas sin ningún tipo de conflicto de ello depende la integración de todo este personal y el buen funcionamiento dentro de las mismas.

A su vez, es preciso que prevalezca una forma de entendimiento entre las partes o sea, entre el directivo y los demás miembros que conforman la organización, tomando siempre en cuenta que cada una de las personas son seres únicos con una manera desigual de ver y de hacer las cosas de allí, la necesidad de respetar la individualidad de cada una de las personas que conforman la estructura organizativa de las instituciones, siempre aunado a una buena gerencia de parte del directivo en mejora del ambiente de trabajo.

\section{Referencias}

Alvarado, O. (2003). Gerencia y Marketing Educativo. Lima, Perú: Universidad Alas Peruanas UDEGRAF S.A.

Calero, M. (2005). Hacia la Excelencia de la Educación. Lima: San Marcos. 306, 215. (B.N.P.). 379.1 C23.

Chiavenato, I. (2000). Introducción a la Teoría General de la Administración. Quinta Edición. Editorial McGraw-Hill. México.

García-Ayuso, M. (2003). Meritum Project's: Guidelines for Managing and Reporting on Intangibles. Ponencia presentada en International research seminar intangibles and intellectual capital: emerging issues in company valuation, reporting and management, Ferrara. 
Gardner, H. (1986). Multiple Intelligences go to School: Educational Implications of the Theory of Multiple Intelligences. Educational Researcher, 18, 4-10.

Hunter, J. (2001), La Paradoja: Un relato sobre la verdadera esencia del Liderazgo. Empresa Activa. Barcelona 2001. Di Ana Mª Hernández Fernández. Manual de Técnicas de la Investigación para estudiantes de ciencias sociales. Colegio de México.

Matos, V. (2009). Gerencia y Liderazgo. Cuadro Comparativo Universidad "Fermín Toro" Gerencia Empresarial. Barquisimeto, estado Lara.

Normas para los colaboradores (2009). La Revista Venezolana de Educación. Educere, Año 13, Número 44. Mérida, Venezuela.

Ortiz, F. y García, M. (2002). Metodología de la Investigación: el Proceso y sus Técnicas. Ed. Limusa.

Ribeiro, L. (2003). La Comunicación Eficaz. Ediciones Urano. Barcelona, España.

Robbins, S. (2004), Comportamiento Organizacional. Décima Edición México: Pearson.

UNESCO (2013). Taller sobre Liderazgo Directivo Escolar en América Latina y el Caribe. Santiago de Chile: Paz Portales, Coordinadora del Programa de OREALC-UNESCO.

Zaldívar, D. (2004). Relaciones Interpersonales. s.e. 


\section{César Orlando Becerra Ramírez \\ e-mail: cesarbecerra70@gmail.com}

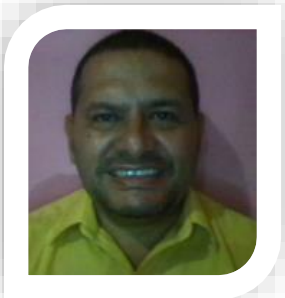

Natural de Valera Estado Trujillo, actualmente cursante del IV semestre del Doctorado en Educación Universidad Nacional Experimental "Rafael María Baralt", extensión Valera, Especialista en Planificación y Evaluación Educacional de la Universidad Valle De Momboy Valera (2004), Licenciado en Educación Mención Biología de la Universidad de Los Andes (2001).

Actualmente se desempeña como coordinador Pedagógico de los 2dos años de Educación Media general con 21 años de servicio (8 años como Docente Integral y 13 años en Educación Media General), de la Unidad Educativa Barrio Nuevo, ubicada en Valera Estado Trujillo. Me desempeñé como: coordinador de Educación rural, zona Educativa Trujillo (2004), Coordinador intermisiones Trujillo (Misión Robinson, Misión Ribas, Misión Sucre, 2004), Coordinador Municipal CEMAMEC (2005), Coordinador institucional PAE (2013), Coord seccional 2do año de Educación media general (2014), Coordinador Sociedad Bolivariana (2015).

El contenido de este manuscrito se difunde bajo una Licencia de Creative Commons ReconocimientoNoComercial-Compartirlgual 4.0 Internacional 